\title{
Un objet d'études : les associations confessionnelles à vocation sociopolitique
}

\section{François Mabille}

\section{(2) OpenEdition}

1 Journals

Édition électronique

URL : http://journals.openedition.org/assr/2163

DOI : 10.4000/assr.2163

ISSN : $1777-5825$

Éditeur

Éditions de l'EHESS

\section{Édition imprimée}

Date de publication : 1 avril 2004

Pagination : 41-45

ISBN : 2-222-96746-5

ISSN : 0335-5985

\section{Référence électronique}

François Mabille, «Un objet d'études : les associations confessionnelles à vocation sociopolitique», Archives de sciences sociales des religions [En ligne], 126 | avril - juin 2004, mis en ligne le 17 novembre 2005, consulté le 03 mai 2019. URL : http://journals.openedition.org/assr/2163 ; DOI : 10.4000/ assr. 2163 


\title{
UN OBJET D'ÉTUDES : LES ASSOCIATIONS CONFESSIONNELLES À VOCATION SOCIOPOLITIQUE
}

\author{
À propos de : \\ BEN NEFISSA (Sarah), éd., Pouvoirs et associations \\ dans le monde arabe. Paris, CNRS Éditions, 2002, \\ $179 \mathrm{p}$. \\ MAURER (Catherine), Caritas. Un siècle de charité \\ organisée en Alsace. Strasbourg, Éditions du Signe, \\ 2003, 157 p. (bibliogr., illustr.). \\ MARTINEAUD (Jean-Pierre), Les Ordres religieux \\ dans les hôpitaux de Paris. Les congrégations hospita- \\ lières dans les hôpitaux de l'Assistance publique à Paris : \\ des fondations à la lä̈cisation Paris, L'Harmattan, \\ 2002, 430 p. (Préface d'Alain Cordier) (bibliogr.) (coll. \\ « Histoire de Paris »). \\ CURTIS (Sarah. A.), L'Enseignement au temps des \\ congrégations. Le diocèse de Lyon (1801-1905). Lyon, \\ Presses universitaires de Lyon, 2003, 279 p. (Préface \\ de Claude Langlois, traduit par Hervé Daniélou) \\ (bibliogr.).
}

L'analyse des sociétés politiques et des relations internationales contemporaines a été marquée ces dernières années par l'émergence d'un nouveau domaine d'étude : celui des acteurs associatifs, également appelés à l'échelle internationale acteurs dits «non gouvernementaux », ou encore «transnationaux »: les ONG.

En trente ans, l'intérêt accordé aux Organisations Non Gouvernementales présentes sur la scène internationale sous cette appellation depuis la Charte des Nations Unies et son article 71, a profondément évolué. Faut-il le rappeler : les ONG ont longtemps été considérées comme existant à la marge du système international. Marcel Merle, dans un article célèbre publié il y a plus de 20 ans, hésitait encore à les classer parmi les acteurs des relations internationales (« Le statut international des organisations non gouvernementales» in: Les acteurs dans les 
relations internationales, Paris, Economica, 1986). Les politistes ont ainsi réévalué l'approche internationale et surtout transnationale. L'ouvrage de Risse-Kappen (Bringing the Transnational Relations Back-in, Cambridge, Cambridge University Press, 1995), Transnational Relations pouvait donc présenter en 1995 une synthèse théorique des différentes approches possibles pour analyser ce phénomène. RisseKappen mettait notamment en avant deux approches théoriques pour expliquer les activités et capacités de ces ONG : le rôle des «domestic structure » (formes organisationnelles et normatives qui enchâssent État et société), et l'institutionnalisation internationale (le degré d'institutionnalisation perçu à travers les accords, « régimes », organisations qui régulent un domaine particulier d'activités).

Les études menées ces dernières années se situent à l'entrecroisement de plusieurs problématiques : problématiques générales comme celle de la compréhension de la mondialisation et de l'érosion de la souveraineté de l'État ou encore, thème proche, analyse de la mondialisation économique et, en contrepoint, réflexion sur la contestation transnationale dans le sillage de Seattle comme dans le dernier ouvrage d'Ulrich Beck : Pouvoir et contre-pouvoir à l'ère de la mondialisation(1) dont le chapitre 6 est entièrement consacré aux «stratégies des mouvements de la société civile»; d'où également des travaux sur l'émergence d'une opinion publique internationale ou d'une "société civile internationale », thèmes qui ont pu être abordés autrement par le prisme, par exemple des mutations du militantisme; problématiques plus spécifiques encore comme l'émergence du droit d'ingérence humanitaire dans le système politique international d'après guerre froide ; études fondées enfin sur la place de ces nouveaux acteurs au sein des politiques étrangères des États, dans le cadre d'une réarticulation par subsidiarité ou instrumentalisation de certains segments des politiques étrangères, tant à l'échelle nationale qu'internationale; ou encore celle de la privatisation de la diplomatie avec l'émergence d'acteurs privés.

Toutes ces analyses manifestent qu'associations et ONG sont de plus en plus impliquées dans les politiques et institutions nationales et internationales. La fin de la guerre froide a facilité leur montée en puissance, notamment à l'occasion des conférences internationales qui se sont tenues depuis le début des années quatrevingt. Mais la mondialisation produit également ses effets, notamment avec la crise de l'État-Providence. C'est donc dans une perspective globale de réarticulation des relations entre politique, économie et religieux, et spécialement de leur reconfiguration en régime libéral qu'il convient de pousser l'analyse et ce sans négliger une mise en perspective socio-historique. Dans le domaine politique, l'émergence des associations et ONG concerne tout à la fois ce que Bruno Jobert a appelé la «métamorphose de la régulation étatique » et donc un déplacement des frontières du privé et du public en même temps qu'un réagencement des modalités d'intervention étatique ; mais cette émergence - ou nouvelle attention portée au phénomène associatif religieux dans ses rapports à l'autorité publique - touche également de manière générale ce que Pierre Rosanvallon nomme le «modèle politique français », à savoir un «laboratoire exemplaire de la tension moderne entre le particulier et le général, les corps intermédiaires et l'État ».

Dans le domaine économique, l'approche des ONG comme acteurs producteurs de charité et de solidarité - thème du colloque organisé il y a quelques années à

(1) Paris, Aubier, 2003. 
Strasbourg par Gilbert Vincent - oblige à s'interroger sur la spécificité de ce type d'acteurs et notamment ceux à référence confessionnelle comme agents économiques, notamment dans des registres variés de régimes libéraux : la comparaison entre les ONG américaines sollicitées par G.W. Bush et leur place en Europe dans le secteur de l'économie sociale et solidaire, pourraient être fructueuse, ainsi qu'une réflexion sur les conséquences de la crise de l'État-Providence sur ces ONG.

D'où provient cette émergence du fait associatif national, international et transnational ? Comment interpréter cette montée en puissance des associations et ONG, comment comprendre leur action au niveau des Etats et sur la scène internationale ?

Plusieurs ouvrages récemment parus constituent des apports non négligeables. Avec Pouvoirs et associations dans le monde arabe, Sarah Ben Néfissa poursuit une réflexion essentielle menée par comparaison. On retrouve dans les contributions présentées des analyses proches de celles évoquées précédemment, notamment en ce qui concerne le lien entre l'essor des sphères associatives et la crise économique et du lien social.

Mais l'ouvrage, ambitieux, est par ailleurs d'un double apport.

a) Dans une perspective historique menée par comparaison, les auteurs montrent très clairement la différence avec le cas français par exemple. Dans les pays arabes, les regroupements associatifs qui datent de la fin du XIX ${ }^{\mathrm{e}}$ ne sont pas liés à la dissolution sociale mais à l'occupation coloniale et donc à la question nationale. Les élites qui y contribuent cherchent certes à résoudre également un problème social existant, mais interprété comme "source de l'arriération de leur société et qui aurait rendu possible la domination étrangère » (p. 15). Enfin, cette mobilisation est formulée en terme largement religieux. Dès lors, l'indépendance des États colonisés signifie le début de la prise en charge politique et sociale de questions jusque-là traitées par ces faux «corps intermédiaires » ou opposants semi-déclarés, et donc sur le court terme, un déclin des associations. Il faut attendre la fin de la rente pétrolière et donc l'apparition de la crise économique qui touche directement la légitimité de ces États pour que les associations et ONG connaissent un nouvel essor, non seulement en raison d'une nouvelle question sociale favorisant des regroupements communautaires à fondement parfois religieux - mais pas uniquement - mais aussi de par leur perception comme espace de recours intermédiaire face au politique : "les associations du monde arabe sont souvent le lieu d'émergence et de construction de notabilités politiques. Cette donnée n'est pas propre aux leaders islamistes. Les associations sont un moyen d'élargir des bases d'influence qui peuvent servir de base électorale et politique. Cet aspect est important quand on connaît les difficultés et le faible poids des partis politiques dans cette région » $(25)$.

b) L'intérêt des contributions est également de montrer comment se joue l'articulation entre l'État et la sphère associative. Ici encore, la perspective comparative est bien menée. On peut encore l'élargir. On retrouve ainsi dans cet ouvrage des constats établis antérieurement pour le continent asiatique dans un ouvrage de Shinichi Shigetoni recensé antérieurement, The States and NGOS-Perspective from Asia, (Singapour, Institute of Southeast Asian Studies, 2002) (cf. François Mabille, Religions et mondialisation: nouvelle configuration, nouveaux acteurs, Archives de Sciences Sociales des religions, 2003, $\mathrm{n}^{\circ}$ 122, pp. 27-30.). Selon les contextes nationaux, c'est bien une relation triangulaire qui se met en place entre le marché, 
l'État et les associations, où la variable politique des associations - construction de notabilité politique et/ou émergence d'une contestation politique - dépend du caractère plus ou moins libéral ou autoritaire du régime en place et où la variable économique, quant à elle, dépend à la fois de l'échec plus ou moins prononcé de l'État distributif et de l'intérêt porté à cette situation par les institutions internationale qui voient, dans les associations et ONG un instrument commode, voire docile, à la fois pour contourner le politique et imposer leur langage international (good governance, accountability, etc.). Le cas palestinien, fort bien analysé, est ici exemplaire.

L'une des questions posée par l'ouvrage porte bien sûr sur la nature confessionnelle de certaines associations. Les problèmes sont bien entendu nombreux en ce domaine, le colloque de l'Association française de Sciences religieuses sur les ONG confessionnelles organisé en février 2004 l'a amplement montré. Ainsi en terme d'identité : que se joue-t-il au niveau d'une identité religieuse dans un militantisme politico-caritatif ? Comment les contacts et actions menées sur le terrain avec différentes populations ou avec des ONG non confessionnelles, ou encore avec les institutions internationales modifient-ils - ou renforcent-ils ? - l'identité religieuse originelle ? Qu'est-ce que la nature de l'organisation confessionnelle nous apprend par exemple sur la culture du groupe ? Comment des valeurs religieuses se concrétisent et informent des modalités d'action spécifiques? Qu'est-ce qui se joue dans l'internationalisation d'une structure confessionnelle? Qu'en est-il de la mission, du prosélytisme ou plus globalement d'une ingérence culturelle? Comment se réarticulent les marqueurs identitaires religieux et nationaux, etc. ?

C'est sur ces questions que deux autres ouvrages apportent des éclairages importants. Catherine Maurer analyse la charité en Alsace de manière particulièrement pertinente, en portant son attention sur un acteur local, la Fédération catholique de charité de la ville de Strasbourg. Cette monographie est très riche d'enseignement sur le catholicisme alsacien certes, mais plus largement sur les catholicismes français et allemand puisque la Fédération, créée en 1898 lorsque l'Alsace était allemande, entre dans le giron catholique français après 1918. Les changements de statuts et d'organisation interne constituent des exemples particulièrement intéressants des différences de cultures nationale et organisationnelle qui affectent les catholicismes et qui ne sont habituellement pas pris en compte dans l'analyse. Surtout, l'ouvrage manifeste comment la Fédération catholique, directement engagée sur le terrain sanitaire et social, a subi la concurrence d'un nouveau modèle, celui de l'Action catholique, à partir des années trente. On est là au cœur d'une question qu'il faudra bien aborder de front dans les recherches historiques : jusqu'à quel point la segmentation de l'Action catholique était-elle - est-elle pertinente ? De quel présupposé relève-t-elle en terme d'analyse et de compréhension du champ social et politique? Quels ont été les effets pervers (au sens bien évidemment sociologique du terme) introduits dans le catholicisme français dans son rapport avec son environnement social et politique ? Ce problème se pose à nouveau lorsque la Fédération intègre le Secours catholique (et devient donc la Caritas - Secours catholique d'Alsace) alors même que le modèle de l'organisation créée par Rodhain est, on le sait, très différent de celui de l'Action catholique. Ce qui se joue porte sur la réelle pertinence sociale d'un catholicisme directement en lien avec les politiques publiques locales, régionales et nationales, sur son insertion dans les dispositifs institutionnels et concerne donc à ce titre trois autres 
problématiques: la concurrence avec les organismes publics qui marginalisent éventuellement ce type d'activité à ressort initialement confessionnel ; la technicisation qui affecte ces questions sanitaires et sociales et qui, par voie de conséquence, induit une professionnalisation ; enfin la question de la laïcité et des relations avec les pouvoirs publics, peu traitée dans cet ouvrage mais présente en filigrane.

Ce sont ces questions qu'aborde également J.-P. Martineaux dans son livre Les Ordres religieux dans les hôpitaux de Paris. On ne reviendra pas ici sur les confirmations apportées par l'ouvrage à une histoire déjà bien connue, à savoir l'apport multiséculaire des ordres religieux dans le domaine de la santé, la lutte initiée après la Révolution française pour reléguer à la marge des acteurs au moment où émerge l'État français simultanément républicain et «État-Providence»; l'étude plus factuelle que problématisante, insiste bien également sur les processus de sécularisation et de laïcisation de ces secteurs et de leurs personnels. L'intérêt de l'ouvrage est sans doute ailleurs, même si tel n'est pas son propos, en permettant de comprendre comment ces ordres - mais on peut également intégrer l'exemple de la Fédération catholique de la charité dans ce schéma - sont confrontés non pas uniquement à un processus de déclin sur le mode de la perte par la confrontation (sécularisation/laïcisation) mais aussi voire surtout à une double transformation : évolution rapide du secteur d'activité concerné - ici la médecine qui repose sur l'émergence de personnels de plus en plus différenciés par leurs métiers, leurs appartenances sociales et donc aussi dans la représentation dont ils sont affectés dans l'imaginaire social - ; changement également du type d'institution sur lequel reposaient ces activités charitables religieuses. Les dernières pages ouvrent la perspective d'une passionnante étude à mener sur la création de nouveaux établissements hospitaliers et de fondations et pas uniquement sur Paris au demeurant. Car, comme le rappelle l'auteur, «le processus de laïcisation des hôpitaux publics entraîna une explosion de fondations privées après 1880 », et souvent confiées à des congrégations religieuses. Qui sait qu'actuellement ces forces ne sont pas que résiduelles en France et qu'elles tentent même, sous l'égide de la Conférence des Supérieures majeures, de s'organiser en fédération aux niveaux français et européen? C'est un vaste champ d'investigation qui porte sur les fondations, les associations et les ONG confessionnelles qui apparaît et obligera sans doute à réévaluer certaines interprétations habituelles des lignes de forces religieuses en France notamment.

En contrepoint, on signalera la très belle thèse de Sarah A. Curtis sur L'Enseignement au temps des congrégations, centré sur le diocèse de Lyon, et qui, à partir de constats historiques identiques, analyse l'exemple du secteur éducatif qui, à l'inverse du secteur hospitalier, a su résister au processus de sécularisation, découplé de celui de la laïcisation.

François MABILLE

Centre d'Études Interdisciplinaires des Faits Religieux EHESS-CNRS 